\title{
On the Structure of the Algebra of Field Operators. II
}

$$
\text { By }
$$

\author{
H. J. Borchers
}

Institut des Hautes Etudes Scientifiques
Bures-sur-Yvette (S. \& O.) France

\begin{abstract}
The global structure analysis of local field operators is reinvestigated.
\end{abstract}
\section{Introduction}

In an earlier paper [1] we investigated the global structure of a given field theory. The assumptions of that paper were that the field theory is given in terms of Wightman functions which fulfil:

1) Wightman functions are tempered distributions,

2) Invariance under the inhomogeneous Lorentz group,

3) Spectrum condition,

4) Local commutativity,

5) Condition of positive definiteness.

Furthermore, there was a hidden assumption which was not explicitly mentioned, namely,

6) For real test functions $f(x)$ the canonical representation of $A(f)$ is essentially self-adjoint and the spectral resolutions of $A(f)$ and $A(g)$ commute if the supports of $f$ and $g$ are spacelike to one another.

In another paper with Zimmermann [2] we studied the question whether 6) can be derived from the assumptions 1)-5). It turned out that 6) can only be derived if certain very restrictive conditions are fulfilled. We gave a trivial counterexample showing that 6 ) is not a consequence of the other assumptions. The additional condition we had to introduce is so restrictive that even the Wick-ordered cube of a free field does not satisfy it. WIghTMAN [3] gave arguments indicating that this field is indeed not self-adjoint. In this special case one can construct ring systems, and $J_{\mathrm{AFFE}}[4]$ has shown that they are local.

Recently ARAKI [5] investigated the global structure of local ring systems and found that such a theory is only irreducible if the Hilbert space contains a single vacuum state when cyclicity is assumed. 
In this paper we want to re-examine this question for local fields without using the condition 6). We will need the following assumptions:

a) Wightman functions are distributions,

b) Invariance under the translation group,

c) Spectrum condition,

d) Local commutativity,

e) Condition of positive definiteness.

In [5] ARAKI used a certain smoothness condition for the spectral measure $d E_{p}$ of the translation group. It turned out that this condition is always fulfilled as a consequence of the conditions a)-e). As our main tool we employ techniques of analytic functions developed in a recent paper [6].

We will assume that the reader is familiar with unbounded operators in a Hilbert space. A short review of this subject can be found in reference [2].

\section{Examples}

In this section we want to discuss some examples of fields which are irreducible, but where the Hilbert space contains more than one vacuum state.

Let $\left\{\mathcal{G}_{1}, U(a), A(x)\right\}$ be a field theory in the Hilbert space $\mathfrak{G}_{1}$ and $U(a)$ the unitary representation of the translation group. Assume there is only one vacuum state in $\mathfrak{G}_{1}$. Denote by $\mathfrak{S}_{1}$ the cyclic domain of the field $A(x)$ in $\mathfrak{G}_{1}$.

Now let $\mathfrak{G}_{0}$ be any Hilbert space and $B$ a symmetric but not selfadjoint operator in $\mathfrak{H}_{0}$. Let $\mathfrak{S}_{0}$ be the subspace of all vectors which are in the domain of every power of $B . \mathfrak{S}_{0}$ will be dense in $\mathfrak{S}_{0}$ and is invariant under the action of $B$. Take now the Hilbert space $\mathfrak{G}_{1} \times \mathfrak{G}_{0}$ and in it the unitary representation of the translation group $U(a) \times \mathbf{1}$. On the domain $\mathfrak{S}_{1} \times \mathfrak{S}_{0}$ we can define a field

$$
C(x)=A(x) \times B .
$$

The new theory will again fulfil all axioms of field theory except possibly cyclicity.

We get a more complicated example if we choose in $\mathfrak{G}_{1}$ fields $A_{1}(x)$, $, \ldots, A_{n}(x)$ which are elements of the commutation class of $A(x)$. In $\mathfrak{G}_{0}$ we choose $n$ operators $B_{1} \ldots B_{n}$ with the properties:

There exists a dense domain $\mathfrak{S}_{0}$ which is invariant under the action of $B_{i}, i=1 \ldots n$. For every vector $\Psi \in \mathfrak{P}_{0}$ we have $B_{i} B_{k} \Psi=B_{k} B_{i} \Psi$, $i, k=1 \ldots n$. Finally, the $B_{i}$ are symmetric operators.

We define now in $\mathfrak{G}_{1} \times \mathfrak{G}_{0}$ the field

$$
C(x)=\sum_{i=1}^{n} A_{i}(x) \times B_{i} .
$$


If we assume that the vacuum state in $\mathfrak{S}_{1}$ is a cyclic vector for the fields $A_{i}(x), i=1 \ldots n$, then we see that an operator $C$ commutes with $C(x)$ only if it is of the form $1 \times b$, where $b$ commutes with all $B_{i}$. Hence the global structure analysis of the field $C(x)$ reduces to the structure analysis of the operators $B_{i}, i=1 \ldots n$.

From the study of the $n$-dimensional moment problem it is well known that there do exist $n$ operators $B_{i}$ which have the above properties and which are not self-adjoint.

What is even worse, NeLson [7] has shown that there exist $n$ such operators having the additional properties that the $B_{i}$ are essentially self-adjoint on the domain $\mathfrak{S}_{0}$, but the spectral projections of $B_{i}$ do not commute with those of $B_{k}$

All our examples have the following features in common:

1) $\mathfrak{G}$ is the direct product of two Hilbert spaces $\mathfrak{S}_{1}$ and $\mathfrak{H}_{0}$.

2) $\mathfrak{G}_{1}$ contains only one vacuum state, i.e. $\mathfrak{G}_{0}$ is isomorphic to the sub-Hilbert space of all invariant vectors in $\mathfrak{H}$.

3) $U(a)$ is of the form $U_{1}(a) \times 1$.

4) Let $P_{0}$ be the projection onto the subspace of all invariant vectors in $\mathfrak{G}$. Then we have a linear positive map $\varphi$ from the polynomial algebra of $C(x)$ into an Abelian algebra,

$$
\varphi(\operatorname{Pol} C(f))=P_{0} \operatorname{Pol} C(f) P_{0} .
$$

5) A bounded operator $C$ commutes with all $C(f)$ if and only if it is of the form $1 \times b$, where $b$ commutes with all $\varphi C(f)$.

In the next section we want to show that every field whose commutant has no center fulfils the properties 1)-5). This result strongly suggests that equation (1) gives the general structure. We do not want to investigate this question. Rather we would like to show that the equivalence of irreducibility and one vacuum for cyclic fields fails only because there exist representations of Abelian *-algebras which are not one-dimensional.

\section{Reduction theory}

In this section the details of a reduction theory are worked out.

We will assume that we have a local symmetric field $A(x)$ in a Hilbert space $\mathfrak{F}$ and a unitary representation $U(a)$ of the translation group fulfilling the spectrum condition. $P_{0}$ denotes the projection onto the subspace of all states invariant under the $U(a)$. We assume furthermore the existence of a cyclic domain $\mathfrak{D}_{0} \subset P_{0} \mathfrak{G}$. This means all polynomials of $A(f)$ can be applied to $\mathfrak{G}_{0}$ and the domain

$$
\begin{gathered}
\mathscr{D}\left(\mathfrak{S}_{0}\right)=\text { Linear Span } \times \\
\times\left\{\mathfrak{S}_{0}, A(f) \mathfrak{S}_{0}, A\left(f_{1}\right) A\left(f_{2}\right) \mathfrak{S}_{0}, \ldots, A\left(f_{1}\right), \ldots, A\left(f_{n}\right) \mathfrak{S}_{0}\right\}
\end{gathered}
$$

is dense in $\mathfrak{H}$. Let $A(f)$ be defined only on $\mathscr{D}$. 
From the symmetry assumption it follows that all operators $A(f)$, $A\left(f_{1}\right) A\left(f_{2}\right) \ldots$ are closurable. Define

$$
\hat{\mathfrak{S}}=\bigcap_{f} \mathfrak{S}_{\overline{A(f)}} \bigcap_{f_{1}, f_{2}} \mathfrak{S}_{\overline{A\left(f_{1}\right) A\left(f_{2}\right)}} \cap \cdots .
$$

With this notation we have:

Theorem 1. Let $\hat{\mathfrak{S}}_{0} \equiv \hat{\mathfrak{S}} \cap P_{0} \mathfrak{S}$. Then we have $P_{0} \mathscr{D}\left(\hat{\mathfrak{S}}_{0}\right)=\hat{\mathfrak{S}}_{0}$. Since $\mathscr{D}\left(\mathfrak{S}_{0}\right) \subset \mathscr{D}\left(\hat{\mathfrak{S}}_{0}\right)$ we have that $\hat{\mathfrak{S}}_{0}$ is dense in $P_{0} \mathfrak{S}$.

Proof: Denote by $A(\mathbf{f})$ any polynomial in the $A(f)$. We then have to consider expressions of the form

$$
\left(\Psi, A\left(\mathbf{f}^{*}\right) U(x) A\left(\mathbf{g}^{*} \mathbf{g}\right) U(y) A(\mathfrak{f}) \Psi\right) \text { for } \Psi \in \hat{\mathfrak{D}}_{0} .
$$

We first remark that due to the spectrum condition the operator $U(a)$ can be defined for complex $a$ such that $\operatorname{Im} a \in \mathscr{V}+(\mathscr{V}+$ the forward light-cone). If $\operatorname{Im} a \in \mathscr{V}+$ then

$$
\lim _{\lambda \rightarrow \infty} U(\lambda a)=P_{0}, \operatorname{Im} a \in \mathscr{V}^{+}
$$

in the strong operator topology.

Now according to reference [6], Theorem 2, the expression

$$
\left(\Psi, A\left(\mathfrak{f}^{*}\right) U(x) A\left(\mathrm{~g}^{*} \mathrm{~g}\right) U(y) A(\mathbf{f}) \Psi\right)=F(x, y)
$$

is defined for complex $x$ and $y$ such that $\operatorname{Im} x, \operatorname{Im} y \in \mathscr{V}^{+}$.

We now want to use the assumption that $\mathbf{f}$ and $g$ have compact support to show that (5) is bounded in some part of the forward tube. Let support $\mathbf{f}$ be in the sphere of radius $r_{1}$ and support $g$ in the sphere of radius $r_{2}$. Denote by $\mathscr{G}$ the points which are spacelike to the sphere of radius $r_{1}+r_{2}$.

Then we have for $x \in \mathscr{G}$ and $\operatorname{Im} y \in \mathscr{V}+$ or 0

$$
\begin{aligned}
& \left|\left(\Psi A\left(\mathbf{f}^{*}\right) U(x) A\left(\mathbf{g}^{*} \mathbf{g}\right) U(y) A(\mathfrak{f}) \Psi\right)\right| \leqq \\
\leqq & \left(\Psi A\left(\mathbf{f}^{*}\right) U(x) A\left(\mathbf{g}^{*} \mathbf{g}\right) A\left(\mathbf{g}^{*} \mathbf{g}\right) U(-x) A(\mathfrak{f}) \Psi\right)^{\frac{1}{2}}\left(\Psi, A\left(\mathbf{f}^{*}\right) A(\mathfrak{f}) \Psi\right)^{\frac{1}{2}} .
\end{aligned}
$$

Because of locality and the invariance of $\Psi$ this can be majorised by

$$
\begin{gathered}
\left(\Psi, A\left(\mathfrak{f}^{*}\right) A(\mathfrak{f}) \Psi\right)^{\frac{1}{2}}\left(\Psi A\left(\mathfrak{f}^{*}\right) A(\mathfrak{f}) A\left(\mathfrak{f}^{*}\right) A(\mathfrak{f}) \Psi\right)^{\frac{1}{4}} \times \\
\times\left(\Psi, A\left(\mathbf{g}^{*} \mathbf{g}\right) A\left(\mathbf{g}^{*} \mathbf{g}\right) A\left(\mathbf{g}^{*} \mathbf{g}\right) A\left(\mathbf{g}^{*} \mathbf{g}\right) \Psi\right)^{\frac{1}{4}}
\end{gathered}
$$

From this we learn: expression (5) is uniformly bounded in

$$
x \in \mathscr{G}, \quad \operatorname{Im} y \in \mathscr{V}^{+} \text {or } \quad 0, \quad y \in \mathscr{G}, \quad \operatorname{Im} x \in \mathscr{V}+\quad \text { or } 0 .
$$


Now let $a=\left\{a_{0}, \vec{a}\right\}, a_{0}>0$ be any vector in $\mathscr{G}$. Then

$$
F\left(\lambda_{1}\left(u_{1} a_{0}, \vec{a}\right), \lambda_{2}\left(u_{2} a_{0}, \vec{a}\right)\right)=f_{\lambda_{1}, \lambda_{2}}\left(u_{1}, u_{2}\right)
$$

is uniformly bounded for $\operatorname{Im} \lambda_{1} u_{1}>0$ and $\operatorname{Im} u_{2}=0,\left|u_{2}\right| \leqq 1$ or $\operatorname{Im} \lambda_{2} u_{2}>0$ and $\operatorname{Im} u_{1}=0,\left|u_{1}\right| \leqq 1$ as long as $\lambda_{1}, \lambda_{2}$ are real and $\left|\lambda_{1}\right|,\left|\lambda_{2}\right| \geqq 1$.

From reference [6], Lemma 8 , it follows that $f_{\lambda_{1}, \lambda_{2}}\left(u_{1}, u_{2}\right)$ is uniformly bounded in the domain

$$
\left|u_{1}\right|<1,\left|u_{2}\right|<1, \operatorname{Im} \lambda_{1} u_{1}>0 \operatorname{Im} \lambda_{2} u_{2}>0 .
$$

This implies

$$
\left\|A(\mathrm{~g}) U\left(\lambda\left(u a_{0}, \vec{a}\right)\right) A(\mathbf{f}) \Psi\right\| \quad \text { for } \quad|u|<1, \operatorname{Im} u>0
$$

is bounded for $\lambda>1$. Hence

$$
\lim _{\lambda \rightarrow \infty} A(\mathrm{~g}) U\left(\lambda\left(u a_{0}, \vec{a}\right) A(\mathbf{f}) \Psi\right)
$$

converges weakly since $\left(\Phi, A(\mathbf{g}) U\left(\lambda\left(u a_{0}, \vec{a}\right)\right) A(\mathbf{f}) \Psi\right)$ converges if $\Phi \in \mathfrak{S}_{A^{*}(g)}$.

Since $A(\mathrm{~g})$ is a closed operator we see from this together with equation (4) that $P_{0} A(\mathbf{f}) \Psi$ is in the domain of $A(\mathrm{~g})$ (since the graph of $A(\mathrm{~g})$ is closed), q.e.d.

This theorem permits us to define the operators $A_{0}(\mathbf{f})=P_{0} A(\mathbf{f}) P_{0}$ as operators in $P_{0} \mathfrak{G}$.

These operators are defined on $\hat{\mathfrak{S}}_{0}$ and map $\hat{\mathfrak{S}}_{0}$ into itself. We now prove

Theorem 2. The map $\varphi(\mathbf{f})=P_{0} A(\mathbf{f}) P_{0}$ is a linear positive map into an Abelian algebra.

Proof: The map $P_{0} A(\mathbf{f}) P_{0}$ is clearly linear and positive. Now let $\Psi, \Phi \in \widehat{\mathfrak{S}}_{0}$; we then have to show that the relation

$$
\left(\Psi, A(\mathbf{g}) P_{0} A(\mathfrak{f}) \Phi\right)=\left(\Psi, A(\mathfrak{f}) P_{0} A(\mathbf{g}) \Phi\right)
$$

holds. To this end we consider the function

$f_{\lambda}(u)=\left(\Psi, A(\mathrm{~g}) U\left(\lambda\left(u a_{0}, \vec{a}\right) A(\mathbf{f}) \Phi\right) \quad\right.$ for $\quad|u|<1, \lambda>1,\left(a_{0}, \vec{a}\right) \in \mathscr{G}$

For $\operatorname{Im} u>0$ we have $\lim _{\lambda \rightarrow \infty} f_{\lambda}(u)=\left(\Psi, A(\mathbf{g}) P_{0} A(\mathbf{f}) \Phi\right)$ and for $\operatorname{Im} u<0$ $\lim _{\lambda \rightarrow \infty} f_{\lambda}(u)=\left(\Psi, A(\mathfrak{l}) P_{0} A(\mathrm{~g}) \Phi\right)$.

Since $f_{\lambda}(u)$ is uniformly bounded in $\lambda>0$ and $|u|<1$, we have by Vitali's theorem [8]

$$
\left(\Psi, A(\mathbf{g}) P_{0} A(\mathbf{f}) \Phi\right)=\left(\Psi, A(\mathbf{f}) P_{0} A(\mathbf{g}) \Phi\right), \quad \text { q.e.d. }
$$


Next we have to investigate the commutant of $\{A(\mathbf{f})\}$. To do this we say that a bounded operator $B$ is in the commutant of an unbounded operator $A$ if $B$ and $B^{*}$ commute with $A$.

$$
B \in A^{\prime} \Leftrightarrow A B \supset B A, A B^{*} \supset B^{*} A .
$$

With this definition the commutant of any family of unbounded operators becomes a von Neumann algebra [9].

Theorem 3. Every element of $\{A(\mathbf{f})\}^{\prime}$ commutes with the translation operators $U(a)$. The map $\varphi(B)=P_{0} B P_{0}$ is a one-to-one map of $\{A(\mathbf{f})\}^{\prime}$ onto $\left\{A_{0}(\mathbf{f})\right\}^{\prime}$.

Proof: Since we have a cyclic set of invariant vectors we find by an argument given in Theorem 4 of reference [1] that every element of $\{A(\mathbf{f})\}^{\prime}$ commutes with $U(a)$.

This in particular implies that $P_{0}$ commutes with $\{A(\mathbf{f})\}^{\prime}$. Since $\hat{\mathfrak{S}}_{0}$ is cyclic for $\{A(\mathbf{f})\}$, it is separating for $\{A(\mathbf{f})\}^{\prime}$. This implies $\varphi(B)=P_{0} B P_{0}$ is a one-to-one map of $\{A(\mathbf{f})\}^{\prime}$ into $\left\{A_{0}(\mathbf{f})\right\}^{\prime}$. Let now $B_{0} \in\left\{A_{0}(\mathbf{f})\right\}^{\prime}$. Define $B$ by the relation

$$
B \sum A\left(\mathfrak{f}_{i}\right) \Psi_{i}=\sum A\left(\mathbf{f}_{i}\right) B_{0} \Psi_{i}, \quad \Psi_{i} \in \hat{\mathfrak{D}}_{0} .
$$

If now $B_{0}>0$, we have

$$
\begin{aligned}
\left(\sum A\left(\mathfrak{f}_{j}\right) \Psi_{j}, B \sum A\left(\mathfrak{f}_{i}\right) \Psi_{i}\right) & =\left(\sum A\left(\mathfrak{f}_{j}\right) \Psi_{j}, \sum A\left(\mathbf{f}_{i}\right) B_{0} \Psi\right) \\
& =\left(\sum A\left(\mathbf{f}_{j}\right) B_{0}^{\frac{1}{2}} \Psi_{j}, \sum A\left(\mathfrak{f}_{i}\right) B_{0}^{\frac{1}{2}} \Psi_{i}\right) \geqq 0 .
\end{aligned}
$$

This implies that if $B_{0}$ is a self-adjoint operator then

$$
\begin{aligned}
\text { upper bound of } B & =\text { upper bound of } B_{0} \\
\text { and lower bound of } B & =\text { lower bound of } B_{0} .
\end{aligned}
$$

Hence $B$ is a continuous operator. Since $B$ clearly commutes with $A(\mathbf{f})$, we have that the map $\varphi$ is onto, q.e.d.

The operators $A_{0}(\mathbf{f})=P_{0} A(\mathbf{f}) P_{0}$ are defined in the Hilbert space $P_{0} \mathfrak{H}$. Now we want to define operators $A_{1}(\mathfrak{f})$ in the Hilbert space $\mathfrak{G}$ with domain of definition $\mathscr{D}\left(\hat{\mathfrak{D}}_{0}\right)$.

$$
A_{\mathbf{1}}(\mathbf{f}) \sum A\left(\mathbf{g}_{i}\right) \Psi_{i}=\sum A\left(\mathbf{g}_{i}\right) A_{0}(\mathfrak{f}) \Psi_{i}, \quad \Psi_{i} \in \hat{\mathfrak{D}}_{0} .
$$

$A_{1}(\mathfrak{I})$ is defined on $\mathscr{D}\left(\hat{\mathfrak{S}}_{0}\right)$ and is clearly linear. We have to show that $A_{1}(\mathbf{f})$ us single-valued. Assume

Then

$$
\sum A\left(\mathrm{~g}_{i}\right) \Psi_{i}=0 .
$$

$$
U(\vec{x}) A(\mathfrak{f}) U(-\vec{x}) \sum A\left(\mathbf{g}_{i}\right) \Psi_{i}=0 .
$$


Taking now the limit $\vec{x} \rightarrow \infty$ we get

$$
\begin{aligned}
0 & =\lim _{\vec{x} \rightarrow \infty} U(x) A(\mathbf{f}) U(-\vec{x}) \sum A\left(\mathbf{g}_{i}\right) \Psi_{i}=\lim _{\vec{x} \rightarrow \infty} \sum A\left(\mathbf{g}_{i}\right) U(x) A(\mathbf{f}) \Psi_{i} \\
& =\sum A\left(\mathbf{g}_{i}\right) A_{0}(\mathbf{f}) \Psi_{i}=A_{\mathbf{1}}(\mathbf{f}) \sum A\left(\mathbf{g}_{i}\right) \Psi_{i} .
\end{aligned}
$$

Thus $A_{1}(\mathbf{f})$ is single-valued.

From the definition of $A_{1}(\mathfrak{f})$ it follows immediately that $A_{1}(\mathbf{f})$ is closurable and that $A_{1}(\mathbf{f})$ commutes with the representation $U(a)$ of the translation group. This implies $A_{1}(\mathbf{f})$ commutes with $P_{0}$. Define now the ring generated by $\left\{A_{1}(\mathbf{f})\right\}$ and $\{A(\mathrm{~g})\}^{\prime}$ as the double commutant of the union of these sets.

$$
\mathfrak{R}\left(\left\{A_{1}(\mathbf{f})\right\}, \quad\{A(\mathrm{~g})\}^{\prime}\right)=\left[\left\{A_{1}(\mathbf{f})\right\} \cup\{A(\mathrm{~g})\}^{\prime}\right]^{\prime \prime} .
$$

With this notation we obtain:

Lemma 4. $\Re\left(\left\{A_{1}(\mathbf{f})\right\},\{A(\mathrm{~g})\}^{\prime}\right)$ is a von Neumann algebra of Type $I$. The center of this ring is identical with the center of $\{A(\mathbf{g})\}^{\prime}$.

Proof: The domain $\hat{\mathfrak{D}}_{0}$ is separating for the set $\left\{A_{1}(\mathbf{f})\right\}$ as well as for $\{A(\mathrm{~g})\}^{\prime}$. From this it follows that $P_{0} \mathfrak{G}$ is a cyclic subspace for $\left[\left\{A_{1}(\mathfrak{f})\right\} \cup\right.$ $\left.\cup\{A(\mathrm{~g})\}^{\prime}\right]^{\prime}$. Hence $P_{0} \mathfrak{G}$ is separating for $\mathfrak{R}\left(\left\{A_{1}(\mathfrak{f})\right\}, \quad\{A(\mathrm{~g})\}^{\prime}\right) \equiv \mathfrak{R}$. Since $P_{0}$ commutes with $\Re$, we have that the central support of $P_{0}$ is equal to 1 . Consider the ring $P_{0} \Re$ in $P_{0} \mathfrak{g}$. From Theorem 3 it follows $P_{0} \Re$ is of Type $\mathrm{I}$ and Center of $P_{0} \Re=$ Center of $P_{0}\{A(\mathrm{~g})\}^{\prime}$. This implies Lemma 4 by a well-known theorem on von Neumann algebras [10].

In the following investigation we want to assume that $\{A(\mathrm{~g})\}^{\prime}$ is a factor. The general situation can be derived from this by making a decomposition with respect to the center. How to handle the reduction theory for families of closed unbounded operators has been studied by Nussbaum [11].

Theorem 5. Assume $\{A(\mathrm{~g})\}^{\prime}$ is a factor. Then the Hilbert space $\mathfrak{G}$ can be written as a direct product $\mathfrak{G}_{1} \times \mathfrak{G}_{0}$ such that $U(a)$ is of the form $U_{1}(a) \times 1$. $\mathfrak{G}_{1}$ contains only one vacuum state.

Proof: If $\{A(\mathrm{~g})\}^{\prime}$ is a factor, then $\mathfrak{R}\left(\left\{A_{1}(\mathfrak{f})\right\},\{A(\mathrm{~g})\}^{\prime}\right) \equiv \mathfrak{R}$ is a factor of Type I (Lemma 4). This implies we can write $\mathfrak{S}$ in the form $\mathfrak{G}_{1} \times \mathfrak{G}_{0}$ such that $\mathfrak{R}=\mathbf{1} \times \mathscr{L}\left(\mathfrak{G}_{0}\right)[12]$. $\left(\mathscr{L}\left(\mathfrak{G}_{0}\right)\right.$ denotes the ring of all bounded operators in $\left.\mathfrak{G}_{0}\right)$. Since $U(a)$ is in the commutant of $\mathfrak{R}$ we can write $U(a)$ in the form $U_{1}(a) \times 1$. From the definition of $\Re$ and Theorem 3 it follows that $P_{0} \Re$ restricted to $P_{0} \mathfrak{G}$ coincides with the ring of all bounded operators in $P_{0} \mathfrak{g}$. This means $P_{0}$ is a minimal projection in $\mathfrak{R}^{\prime}$. Or $P_{0}=P_{01} \times 1$ where $P_{01}$ maps onto a one-dimensional space in $\mathfrak{S}_{1}$. This proves the theorem.

We have shown that the most general field having a cyclic subspace of invariant states is the direct integral of fields fulfilling the properties 1)-5) listed in the last section. In particular this implies that for a 
field defined in terms of Wightman functions the Abelian algebra $\varphi(A(\mathbf{f}))$ has the cyclic vector $\Omega$ as a cyclic vector in the Hilbert space $P_{0} \mathfrak{g}$. If $A(\mathbf{f})$ is an irreducible field, than the Hilbert space contains exactly one vacuum state if the representation of the Abelian algebra $\varphi(A(\mathbf{f}))$ is one-dimensional.

\section{References}

[1] Borchers, H. J.: Nuovo Cimento 24, 214 (1962).

[2] 一, and W. ZimmermanN: Nuovo Cimento 31, 1047 (1964).

[3] Wightman, A. S.: Private communication.

[4] JAFFE, A.: Entire Functions of the Free Field, preprint.

[5] ARAKI, H.: On the Algebra of All Local Observables, preprint.

[6] Borchers, H. J.: Nuovo Cimento 33, 1600 (1964).

[7] Nelson, E.: Ann. Math. 70, 572 (1959).

[8] Titchmarsh, E. C.: The Theory of Functions. 5.21. Oxford: University Press 1939.

[9] Stone, M. H.: J. Indian Math. Soc. 15, 155 (1951).

[10] Dixmier, J.: Les Algèbres d'Opérateurs dans l'Espace Hilbertien. Chap. I, $\S 8$, Théorème 1. Paris: Gauthier-Villars 1957.

[11] Nussbaum, A. E.: Duke Math. J. 31, 33 (1964).

[12] Dixmier, J.: loc. cit. [10], Chap. I, § 8, Corollaire 3.

(Received December 1, 1964) 